\title{
NONPARAMETRIC ANALYSIS OF RANDOMIZED EXPERIMENTS WITH MISSING COVARIATE AND OUTCOME DATA
}

\author{
by \\ Joel L. Horowitz \\ Department of Economics \\ University of Iowa \\ Iowa City, IA 52242 \\ and \\ Charles F. Manski \\ Department of Economics and Institute for Policy Research \\ Northwestern University \\ Evanston, IL 60208
}

Revised: June 1999

\begin{abstract}
Analysis of randomized experiments with missing covariate and outcome data is problematic because the population parameters of interest are not identified unless one makes untestable assumptions about the distribution of the missing data. This paper shows how population parameters can be bounded without making untestable distributional assumptions. Bounds are also derived under the assumption that covariate data are missing completely at random. In each case the bounds are sharp; they exhaust all of the information that is available given the data and the maintained assumptions. The bounds are illustrated with applications to data obtained from a clinical trial and data relating family structure to the probability that a youth graduates from high school.
\end{abstract}

Key Words: Identification, attrition, bounds

We thank William G. Henderson, Domenic Reda, and David Williams of the Edward Hines, Jr., Hospital, U.S. Department of Veterans Affairs Cooperative Studies Program Coordinating Center, Hines IL, for providing and helping us to interpret the clinical-trial data analyzed in this paper. The Department of Veterans Affairs retains ownership of the data. The views expressed in this paper are those of the authors and are not necessarily endorsed by the Department of Veterans Affairs. We thank Mark Manuszak and participants in the Santa Fe Institute's conference on Inferential Problems in the Analysis of Treatment Effects for comments on earlier versions of the paper. The research of Joel L. Horowitz was supported in part by NSF grant SBR-9617925. The research of Charles F. Manski was supported in part by NSF grant SBR-9722846. 


\section{NONPARAMETRIC ANALYSIS OF RANDOMIZED EXPERIMENTS WITH MISSING COVARIATE AND OUTCOME DATA}

\section{INTRODUCTION}

This paper is concerned with nonparametric analysis of randomized experiments with observational problems. The existing literature on this subject is concerned mainly with the interpretation of experiments in which some subjects do not comply with assigned treatments. In general, average treatment effects are not identified when there is noncompliance, but they can be bounded (Manski 1989, 1990; Robins 1989, Balke and Pearl 1997). The identifiability of treatment effects within the subpopulation of persons who comply with treatment has been also studied (Bloom 1984; Angrist, Imbens, and Rubin 1996). Manski (1997) studied a converse problem in which subjects comply with treatment but the problem of interest is to predict outcomes in real world settings with imperfect compliance.

Our concern here is the interpretation of experiments with missing covariate and outcome data. Observational problems at the point of randomization sometimes prevent experimenters from collecting complete data on covariates of interest. Observational problems following randomization (e.g., attrition from a clinical trial) sometimes prevent experimenters from collecting complete outcome data. We suppose that outcomes are binary. The extension to more general outcomes can be carried out by using methods like those of Horowitz and Manski (1998) but is beyond the scope of this paper.

Section 2 characterizes the inferences on population parameters of interest that are possible without making untestable assumptions about the distribution of the missing data. The inferences take the form of bounds on population parameters. The bounds are sharp; that is, they exhaust the information that is available from the data. Moreover, the bounds are continuous functions of the probability that an observation is complete (non-missing) and are informative whenever the probability of a complete observation exceeds zero. We also report tighter bounds obtained under the assumption that covariate data 
are missing completely at random (MCAR), with no assumption imposed on the process generating missing outcome data.

Section 3 illustrates the theoretical results by applying them to data obtained in a U.S. Department of Veterans Affairs (DVA) clinical trial of treatments for hypertension (Materson, et al. 1993). A further illustration is presented in Section 4, which revisits a study of the effect of family structure on the probability that a child graduates from high school (Manski, Sandefur, McLanahan, and Powers 1992). Section 5 presents concluding comments. The proofs of theorems are in the Appendix.

To focus attention on the problem of missing covariates and outcomes, we abstract from other observational problems, including noncompliance. Note, however, that when a subject does not comply with the assigned treatment, the experimenter observes an outcome, albeit for a treatment other than the one assigned. When outcome data are missing, the experimenter observes no outcome at all. Therefore, the bounds derived in Section 2 apply, though they may not be sharp, to settings with noncompliance.

The analysis in this paper is deliberately conservative. Our "worst-case" approach of asking what inferences can be made without invoking untestable assumptions contrasts with the "best-case" approaches that dominate the literature on estimation in the presence of missing data. The most common practice is to assume that covariate and outcome data are missing at random. Occasionally, a model of non-random missing data is asserted. Either way, the identification problem is solved and efficiency of estimation becomes the central matter of concern to statisticians. See, for example, Little (1992), Robins, Rotnitzky, and Zhao (1994), and Wang, Wang, Zhao, and Ou (1997).

In our view, it is not sufficient for empirical researchers to know the inferences that can be made if certain distributional assumptions hold. It is also important to characterize the inferences that can be made without imposing these assumptions. The conservative analysis developed here allows the establishment of a domain of consensus among researchers who may hold disparate beliefs about what assumptions are appropriate (Manski 1995). Concern with establishing consensus has motivated some researchers facing 
missing data problems to perform sensitivity analyses in which they entertain a limited range of alternative assumptions about the nature of the missing data (e.g., Little 1995, Little and Yao 1996, Nordheim 1984, Rosenbaum 1995, Rotnitzky, Robins, and Scharfstein 1998). Our approach is a sensitivity analysis that encompasses all non-refutable assumptions about the nature of the missing data.

In an earlier paper (Horowitz and Manski 1998), we studied the problems of missing covariate and outcome data in the analysis of survey response. The present analysis complements and generalizes that work. Whereas Horowitz and Manski (1998) examined certain polar cases of missing data (only outcomes are missing, only covariates are missing, or outcomes and covariates are jointly missing), here we permit mixed patterns of missing covariate and outcome data. Whereas the earlier paper was concerned only with the problem of inference on outcome levels, here we analyze the distinctive problems of inference on the variation of outcomes with treatment and with covariates.

\section{DERIVATION OF THE BOUNDS}

This section derives bounds on population parameters of interest in applications such as those described in Sections 3 and 4. Each member of the population is characterized by the values of a treatment indicator (a positive integer) and a random variable $\left(Y, X, Z_{y}, Z_{x}\right) . \quad Y$ is a binary outcome variable that indicates whether treatment is successful $(Y=1)$ or unsuccessful $(Y=0) . X$ is a covariate. $Z_{y}$ and $Z_{x}$ are indicators of missing data. $Y$ is observed if $Z_{y}=1$ and unobserved if $Z_{y}=0 . X$ is observed if $Z_{x}=1$ and unobserved if $Z_{x}=0$. Data are obtained by assigning individuals randomly to treatments and observing the resulting values of $Y$ and $X$ whenever these are non-missing.

We assume that if $X$ is a vector, then in any data record either all components of $X$ are missing or none are missing. The bounds we derive apply but may not be sharp if different components of $X$ may be missing in different data records. Sharp bounds for this case can be derived using methods similar to those used here but the calculations are beyond the scope of this paper. 
Let $\Pi_{t}(x)$ denote the probability that $Y=1$ conditional on $X=x$ for an individual who is assigned randomly to treatment $t$. Our aim is to estimate three population quantities: $\Pi_{t}(x), \Pi_{t}(x)-\Pi_{s}(x)$ (the difference between conditional success probabilities under two different treatments), and $\Pi_{t}\left(x_{2}\right)-$ $\Pi_{t}\left(x_{1}\right)$ (the difference between conditional success probabilities with different values of $x$ but the same treatment). Sections 2.1-2.3 derive sharp bounds on these quantities under the assumption that identified features of the population are known with certainty. Finite-sample inference is treated in Section 2.4.

\subsection{Bounds on $\Pi_{t}(x)$ and $\Pi_{t}(x)-\Pi_{s}(x)$}

This section obtains bounds on $\Pi_{t}(x)$ and $\Pi_{t}(x)-\Pi_{s}(x)$ when $X$ is discrete. The data are uninformative about these quantities if $X$ is continuous and some observations of $X$ are missing.

Let $P_{t}($.$) denote the probability of the event in parentheses for an individual who is assigned$ randomly to treatment $t$. For $j, k=0$ or 1 , define $E_{t j k}(x)=P_{t}\left(Y=1 \mid X=x, Z_{x}=j, Z_{y}=k\right), A_{t j k}=P_{t}(Y=$ $\left.1 \mid Z_{x}=j, Z_{y}=k\right), G_{t j k}(x)=P_{t}\left(Z_{x}=j, Z_{y}=k \mid X=x\right), Q_{t j k}(x)=P_{t}\left(X=x \mid Z_{x}=j, Z_{y}=k\right)$, and $p_{t j k}=P_{t}\left(Z_{x}=j, Z_{y}\right.$ $=k$ ). Then

$$
\Pi_{t}(x)=\sum_{j} \sum_{k} E_{t j k}(x) G_{t j k}(x)
$$

Application of Bayes' theorem to $G_{t j k}(x)$ in (1) yields

$$
\Pi_{t}(x)=\sum_{j} \sum_{k} E_{t j k}(x) Q_{t j k}(x) p_{t j k} / \sum_{j} \sum_{k} Q_{t j k}(x) p_{t j k}
$$

For $(j, k) \in\{0,1\}$, the sampling process identifies $E_{t 11}(x), Q_{t 1 k}(x), p_{t j k}$, and $A_{t 01}$. It does not identify $Q_{t 0 k}(x), E_{t j 0}(x)$, or $E_{t 01}(x)$. These quantities can have any values in $[0,1]$. However,

$$
A_{t 01}=E_{t 01}(x) Q_{t 01}(x)+B_{t}(x)\left[1-Q_{t 01}(x)\right],
$$


where $B_{t}(x)=P_{t}\left(Y=1 \mid X \neq x, Z_{x}=0, Z_{y}=1\right) . \quad B_{t}(x)$ is not identified and can have any value in $[0,1]$. Therefore, it follows from (3) that

$$
\max \left\{0, \frac{A_{t 01}-\left[1-Q_{t 01}(x)\right]}{Q_{t 01}(x)}\right\} \leq E_{t 01}(x) \leq \min \left[1, \frac{A_{t 01}}{Q_{t 01}(x)}\right]
$$

These bounds are sharp (Horowitz and Manski 1995, Corollary 1.2). Therefore, sharp bounds on $\Pi_{t}(x)$ can be obtained by minimizing and maximizing the right-hand side of (2) with respect to the unidentified quantities subject to $0 \leq Q_{t 0 k}(x) \leq 1,0 \leq E_{t j 0}(x) \leq 1(j, k=0,1)$, and (4). The result is:

\section{Theorem 1: Define}

$$
\begin{aligned}
& D_{t}(x)=\sum_{k=0}^{1} Q_{t 1 k}(x) p_{t 1 k}+p_{t 00}+\left(1-A_{t 01}\right) p_{t 01} \\
& R_{t}(x)=\sum_{k=0}^{1} Q_{t 1 k}(x) p_{t 1 k}+p_{t 00}+A_{t 01} p_{t 01}
\end{aligned}
$$

$S_{t}(x)=E_{t 11}(x) Q_{t 11}(x) p_{t 11}+Q_{t 10}(x) p_{t 10}+p_{t 00}+A_{t 01} p_{t 01}, \quad L_{t}(x)=E_{t 11}(x) Q_{t 11}(x) p_{t 11} / D_{t}(x), \quad$ and $U_{t}(x)=S_{t}(x) / R_{t}(x)$. Then sharp bounds on $\Pi_{t}(x)$ are $L_{t}(x) \leq \Pi_{t}(x) \leq U_{t}(x)$.

A necessary and sufficient condition for the bounds in Theorem 1 to be informative (that is, to satisfy $\left.U_{t}(x)-L_{t}(x)<1\right)$ is $Q_{t 11}(x)>0$ and $p_{t 11}>0$. In other words, the bounds are informative if the probability of a complete observation with $X=x$ exceeds zero.

Now let $L_{s}(x)$ and $U_{s}(x)$ be the quantities obtained from $L_{t}(x)$ and $U_{t}(x)$ by replacing $t$ with $s$. Sharp bounds on $\Pi_{s}(x)$ are $L_{s}(x) \leq \Pi_{s}(x) \leq U_{s}(x)$. Because individuals are assigned randomly to treatments, there are no further constraints on $\Pi_{t}(x)$ and $\Pi_{s}(x)$. Therefore, sharp bounds on $\Pi_{t}(x)-\Pi_{s}(x)$ are as stated in the following corollary to Theorem 1:

Corollary 1.1: Let $s \neq t$. The inequality $L_{t}(x)-U_{s}(x) \leq \Pi_{t}(x)-\Pi_{s}(x) \leq U_{t}(x)-L_{s}(x)$ gives sharp bounds on $\Pi_{t}(x)-\Pi_{s}(x)$. 
These bounds are informative (that is, the difference between the lower and upper bounds is strictly less than 2) whenever $Q_{t 11}(x)>0, Q_{s 11}(x)>0, p_{t 11}>0$, and $p_{s 11}>0$.

2.2 Bounds on $\Pi_{t}\left(x_{2}\right)-\Pi_{t}\left(x_{1}\right)$

This section derives sharp bounds on $\Pi_{t}\left(x_{2}\right)-\Pi_{t}\left(x_{1}\right)$. It follows from (2) that

$$
\Pi_{t}\left(x_{2}\right)-\Pi_{t}\left(x_{1}\right)=\frac{\sum_{j} \sum_{k} E_{t j k}\left(x_{2}\right) Q_{t j k}\left(x_{2}\right) p_{j k}}{\sum_{j} \sum_{k} Q_{t j k}\left(x_{2}\right) p_{t j k}}-\frac{\sum_{j} \sum_{k} E_{t j k}\left(x_{1}\right) Q_{t j k}\left(x_{1}\right) p_{j k}}{\sum_{j} \sum_{k} Q_{t j k}\left(x_{1}\right) p_{t j k}} .
$$

In addition,

$$
A_{t 01}=E_{t 01}\left(x_{2}\right) Q_{t 01}\left(x_{2}\right)+E_{t 01}\left(x_{1}\right) Q_{t 01}\left(x_{1}\right)+B_{t}\left(x_{1}, x_{2}\right)\left[1-Q_{t 01}\left(x_{2}\right)-Q_{t 01}\left(x_{1}\right)\right]
$$

where $B_{t}\left(x_{2}, x_{1}\right)=P_{t}\left(Y=1 \mid X \neq x_{1}, X \neq x_{2}, Z_{x}=0, Z_{y}=1\right)$. The sampling process does not identify $B_{t}\left(x_{2}, x_{1}\right)$ or the unidentified quantities described in Section 2.1. Each unidentified quantity can have any value in $[0,1]$. There are, however, additional relations among these quantities that must be maintained. These are:

$$
\begin{aligned}
& Q_{t 01}\left(x_{2}\right)+Q_{t 01}\left(x_{1}\right) \leq 1, \\
& Q_{t 00}\left(x_{2}\right)+Q_{t 00}\left(x_{1}\right) \leq 1, \\
& A_{t 01} \geq E_{t 01}\left(x_{2}\right) Q_{t 01}\left(x_{2}\right)+E_{t 01}\left(x_{1}\right) Q_{t 01}\left(x_{1}\right),
\end{aligned}
$$

and

$$
A_{t 01} \leq E_{t 01}\left(x_{2}\right) Q_{t 01}\left(x_{2}\right)+E_{t 01}\left(x_{1}\right) Q_{t 01}\left(x_{1}\right)+\left[1-Q_{t 01}\left(x_{2}\right)-Q_{t 01}\left(x_{1}\right)\right]
$$

Inequalities (9) and (10) are obtained from (6) by letting $B_{t}\left(x_{2}, x_{1}\right)$ range over [0,1].

Sharp bounds on $\Pi_{t}\left(x_{2}\right)-\Pi_{t}\left(x_{1}\right)$ are obtained by maximizing and minimizing the right-hand side of (5) over the unidentified quantities subject to (7)-(10) and the constraint that unidentified probabilities must be in $[0,1]$. The result is stated in Theorem 2 below. The following notation is used. Define 


$$
\begin{gathered}
a=E_{t 11}\left(x_{2}\right) Q_{t 11}\left(x_{2}\right) p_{t 11}+Q_{t 10}\left(x_{2}\right) p_{t 10}+A_{t 01} p_{t 01}, \quad b=Q_{t 11}\left(x_{2}\right) p_{t 11}+Q_{t 10}\left(x_{2}\right) p_{10}+A_{t 01} p_{t 01}, \\
d=E_{t 11}\left(x_{1}\right) Q_{t 11}\left(x_{1}\right) p_{t 11}, f=Q_{t 11}\left(x_{1}\right) p_{t 11}+Q_{t 10}\left(x_{1}\right) p_{t 10}+\left(1-A_{t 01}\right) p_{t 01}, \\
g(z)=\frac{p_{t 00}(b-a)}{\left(b+p_{t 00} z\right)^{2}}-\frac{p_{t 00} d}{\left[f+p_{t 00}(1-z)\right]^{2}},
\end{gathered}
$$

and

$$
z^{*}=\left\{\begin{array}{l}
1 \text { if } G(0)>0 \text { and } G(1)>0 \\
0 \text { if } G(0)<0 \text { and } G(1)<0 \\
\text { The solution in }[0,1] \text { to } G(z)=0, \text { otherwise. }
\end{array}\right.
$$

Theorem 2: Sharp bounds on $\Pi_{t}\left(x_{2}\right)-\Pi_{t}\left(x_{1}\right)$ are $L_{t}\left(x_{2}, x_{1}\right) \leq \Pi_{t}\left(x_{2}\right)-\Pi_{t}\left(x_{1}\right) \leq U_{t}\left(x_{2}, x_{1}\right)$, where

$$
U_{t}\left(x_{2}, x_{1}\right)=\frac{a+p_{t 00} z^{*}}{b+p_{t 00} z^{*}}-\frac{d}{f+p_{t 00}\left(1-z^{*}\right)},
$$

and $L_{t}\left(x_{2}, x_{1}\right)=-U_{t}\left(x_{1}, x_{2}\right)$.

These bounds are informative whenever $Q_{t 11}\left(x_{1}\right)>0, Q_{t 11}\left(x_{2}\right)>0$, and $p_{t 11}>0$.

\subsection{Bounds when $X$ Is Missing Completely at Random}

The bounds derived in Sections 2.1-2.2 can be tightened if $X$ is missing completely at random (MCAR). The tighter bounds are derived in this section. Formally, $X$ is $\operatorname{MCAR}$ if $P_{t}\left(Z_{x}=j \mid Y=\ell, X=x\right.$, $\left.Z_{y}=k\right)=P_{t}\left(Z_{x}=j\right)$ for all $x$ in the support of $X$ and all $j, k, \ell=0,1$. Lemma 1 states the consequences of $X$ being MCAR that are important for identification.

Lemma 1: If $X$ is MCAR, then

$$
E_{t 1 k}(x)=E_{t 0 k}(x)
$$

and

$$
Q_{t 1 k}(x)=Q_{t 0 k}(x)
$$


for all $x$ in the support of $X$ and $k=0$ or 1 .

Lemma 1 implies that when $X$ is $\operatorname{MCAR}, E_{t 01}(x)$ and $Q_{t 0 k}(x)(k=0,1)$ are identified. Define $H_{t}(x)=P_{t}\left(Z_{y}=1 \mid X=x, Z_{x}=1\right)$. Then, substituting (11) and (12) into (2) yields

$$
\Pi_{t}(x)=E_{t 11}(x) H_{t}(x)+E_{t 10}(x)\left[1-H_{t}(x)\right] .
$$

All quantities on the right-hand side of (13) are identified except $E_{t 10}(x)$, which can have any value in [0,1]. Therefore, we have:

Theorem 3: Let $L_{m t}(x)=E_{t 11}(x) H_{t}(x)$ and $U_{m t}(x)=1-H_{t}(x)+L_{m t}(x)$. If $X$ is MCAR, then sharp bounds on $\Pi_{t}(x)$ are $L_{m t}(x) \leq \Pi_{t}(x) \leq U_{m t}(x)$. Sharp bounds on $\Pi_{t}(x)-\Pi_{s}(x)(s \neq t)$ are $L_{m t}(x)-U_{m s}(x) \leq \Pi_{t}(x)-\Pi_{s}(x) \leq U_{m t}(x)-L_{m s}(x)$.

The bounds on $\Pi_{t}(x)$ are informative whenever $H_{t}(x)>0$. The bounds on $\Pi_{t}(x)-\Pi_{s}(x)$ are informative whenever $H_{t}(x)>0$ and $H_{s}(x)>0$.

The MCAR condition can be used to obtain bounds on $\Pi_{t}\left(x_{2}\right)-\Pi_{t}\left(x_{1}\right)$ that are tighter than those of Theorem 2. Substitution of (11) and (12) into (5) yields a version of $\Pi_{t}\left(x_{2}\right)-\Pi_{t}\left(x_{1}\right)$ in which the only unidentified quantities are $E_{t 10}\left(x_{2}\right)$ and $E_{t 10}\left(x_{1}\right)$. These quantities can have any values in [0,1]. Under MCAR, $\Pi_{t}\left(x_{2}\right)-\Pi_{t}\left(x_{1}\right)$ is maximized (minimized) by setting $E_{t 10}\left(x_{2}\right)=1(0)$ and $E_{t 10}\left(x_{1}\right)=0$ (1). Therefore, the following theorem holds.

Theorem 4: Define $U_{m t}\left(x_{2}, x_{1}\right)=1-\left[1-E_{t 11}\left(x_{2}\right)\right] H_{t}\left(x_{2}\right)-E_{t 11}\left(x_{1}\right) H_{t}\left(x_{1}\right)$ and $L_{m t}\left(x_{2}, x_{1}\right)=$ $-U_{m t}\left(x_{1}, x_{2}\right)$. If $X$ is MCAR, then tight bounds on $\Pi_{t}\left(x_{2}\right)-\Pi_{t}\left(x_{1}\right)$ are $L_{m t}\left(x_{2}, x_{1}\right) \leq \Pi_{t}\left(x_{2}\right)-\Pi_{t}\left(x_{1}\right)$ $\leq U_{m t}\left(x_{2}, x_{1}\right)$

These bounds are informative whenever $H_{t}\left(x_{2}\right)>0$ and $H_{t}\left(x_{1}\right)>0$. In addition, it is not difficult to show that Theorems 3 and 4 hold if $X$ is continuously distributed. Thus, under the MCAR assumption, we 
obtain informative bounds even if $X$ is continuous. As was discussed in Section 2.1, the bounds are uninformative if $X$ is continuous but not assumed to be MCAR.

\subsection{Finite-Sample Inference}

The discussion in Sections 2.1-2.3 assumes that identified features of the joint distribution of $(Y, X$, $\left.Z_{y}, Z_{x}\right)$ are known with certainty. This section treats inference when these features must be estimated from a finite sample of size $n$. We explain how to obtain consistent point estimators of the bounds and joint asymptotic confidence intervals that have known probabilities of containing the population values of both the lower and upper bounds.

First, consider point estimation of the bounds. The population bounds given in Sections 2.1-2.3 are functionals of population probabilities of components of $\left(Y, X, Z_{y}, Z_{x}\right)$. Thus, consistent estimators of these bounds can be obtained by replacing the population probabilities with empirical probabilities.

Now consider a joint confidence interval for a pair of lower and upper bounds. The interval of greatest interest is one that has a known (asymptotic) probability of containing both the lower and the upper bound on an unidentified population feature. The interval obtained here has the form $\left[L_{n}-z_{n}, U_{n}+\right.$ $z_{n \alpha}$ ], where $L_{n}$ and $U_{n}$ are estimates of the lower and upper bounds $L$ and $U$ on an unidentified population feature. The number $z_{n \alpha}$ is chosen so that $P\left(L_{n}-z_{n \alpha} \leq L, U \leq U_{n}+z_{n \alpha}\right)=1-\alpha$ asymptotically.

One way of obtaining $z_{n \alpha}$ is to derive it from an analytic expression for the asymptotic distribution of $L_{n}$ and $U_{n}$. The delta method can be used to show that for each pair $(L, U)$ in Section 2.1-2.3, the estimator $\left(L_{n}, U_{n}\right)$ is asymptotically bivariate normally distributed with mean $(L, U)$ and a covariance matrix that can be estimated consistently. This approach is unattractive, however, because the expressions for the asymptotic covariance matrices are very lengthy and, therefore, tedious to implement.

Asymptotically valid confidence intervals can be obtained more easily by using the bootstrap. This consists of carrying out a Monte Carlo simulation in which the data corresponding to the treatment(s) of interest are sampled randomly with replacement (bootstrap sampling). Each bootstrap sample is used to 
compute a bootstrap estimate of $(L, U)$, denoted $\left(L_{n}{ }^{*}, U_{n}{ }^{*}\right)$, by applying the formulae for $\left(L_{n}, U_{n}\right)$ to the bootstrap sample. By repeated bootstrap sampling, the distribution of $\left(L_{n}^{*}, U_{n}^{*}\right)$ conditional on the data can be estimated with arbitrary accuracy. The estimated distribution is used to find $z_{n \alpha}{ }^{*}$ such that $P^{*}\left(L_{n}{ }^{*}-\right.$ $\left.z_{n \alpha}{ }^{*} \leq L_{n}, U_{n} \leq U_{n}^{*}+z_{n \alpha}{ }^{*}\right)=1-\alpha$, where $P^{*}$ is the probability measure induced by bootstrap sampling conditional on the estimation data. The bootstrap $1-\alpha$ confidence interval for $(L, U)$ is $\left[L_{n}-z_{n}{ }^{*}, U_{n}+\right.$ $\left.z_{n \alpha}{ }^{*}\right]$. It has asymptotic coverage probability $1-\alpha$ (Bickel and Freedman 1981). The bootstrap is used to obtain confidence intervals for bounds in the applications described in Sections 3 and 4.

To check the finite-sample accuracy of the bootstrap procedure, we carried out a Monte Carlo experiment in which we estimated the true coverage probabilities of nominal $95 \%$ confidence intervals for bounds on $\Pi_{t}\left(x_{2}\right)-\Pi_{t}\left(x_{1}\right)$. The distribution of $\left(Y, X, Z_{y}, Z_{x}\right)$ used for the simulations was the empirical distribution of the data in the DVA clinical trial that is described in Section 3. The sample sizes were the same as in the clinical trial. Experiments were carried out with and without assuming that $X$ is MCAR. The empirical coverage probabilities were in the range 0.93 to 0.96 .

\section{AN ILLUSTRATIVE APPLICATION TO A CLINICAL TRIAL}

This section applies the methods of Section 2 to data obtained in a clinical trial of treatments for hypertension that was carried out by the DVA (Materson, et al. 1993; Materson and Reda 1994; Materson, Reda, and Cushman 1995). Patients at 15 DVA hospitals were randomly assigned to one of 6 treatments for hypertension or placebo (treatment 7). The trial had two phases. In the first, the dosage that brought diastolic blood pressure (DBP) below $90 \mathrm{~mm} \mathrm{Hg}$ was determined. In the second, it was determined whether DBP could be kept below $95 \mathrm{~mm} \mathrm{Hg}$ for a long time. Treatment is defined to be successful if DBP $<90 \mathrm{~mm} \mathrm{Hg}$ on two consecutive measurement occasions in the first phase and DBP $\leq 95 \mathrm{~mm} \mathrm{Hg}$ in the second. Treatment is unsuccessful otherwise. The biochemical indicator "renin response" was 
measured at the time of randomization. It has levels "low," "medium," and "high," and may be related to the probability of successful treatment (Freis, Materson, and Flamenbaum 1983).

Let $X$ denote renin response. The problem addressed here is to estimate $\Pi_{t}\left(x_{j}\right)-\Pi_{t}\left(x_{k}\right)(j \neq k)$, and $\Pi_{t}(x)-\Pi_{7}(x)$ for $t \neq 7$ (the treatment effect). Some patients dropped out of the trial before success or failure could be determined, and renin-response is missing for some patients. Therefore, the quantities of interest are not identified. We use the methods of Section 2 to obtain bounds on them. The pattern of missing data is shown in Table 1.

We have obtained bounds on the population quantities of interest without and with the assumption that renin response is MCAR. The MCAR assumption is not testable with the available data but is plausible for reasons that will now be explained. All randomized patients had blood samples taken for the purpose of determining renin response. The samples were shipped to a central laboratory for analysis. Missing data on renin response occurred when samples were damaged or destroyed during shipment. Neither the outcome of a patient's treatment nor his renin response were known at the time of shipment. Thus, it is reasonable to assume that the occurrence of damage and, therefore, $Z_{x}$ are statistically independent of treatment outcome and renin response, at least conditional on the hospital at which the sample originated. A chi-square test failed to reject the hypothesis that $Z_{x}$ is independent of hospital ( $p>$ 0.30). A chi-square test using data from the patients for whom $Z_{y}=1$ failed to reject the hypothesis that $Z_{x}$ is independent of $Y(p>0.5)$. The tests support (but do not imply) the assumption that $Z_{x}$ is independent of the other observed variables.

We have also obtained point estimates of the population quantities of interest using only records with complete data. This amounts to assuming that missing data are ignorable. We have no evidence for or against this assumption. We use it to illustrate the gains in precision of inference that it provides.

The results are shown in Tables 2 and 3. As expected, the point estimates and 95\% confidence intervals for the bounds are narrower with than without the assumption that $X$ is MCAR and narrower still 
when only complete-data records are used. Table 2 shows that the $95 \%$ confidence intervals for the treatment effect conditional on low renin response exclude zero for 5 of the 6 treatments, even when no assumptions are made about the distribution of missing data. For each of these treatments, the hypothesis that treatment is ineffective conditional on low renin response is rejected at the 0.05 level without making untestable assumptions. The $95 \%$ confidence intervals for the treatment effect conditional on medium renin response exclude zero for 2 treatments without the MCAR assumption, 4 treatments with MCAR, and 5 treatments when missing observations of $Y$ and $X$ are assumed to be ignorable. With high renin response, the hypothesis of ineffective treatment is rejected for one treatment with no assumptions about the distribution of the missing data, 3 treatments with MCAR, and 4 treatments when missing observations of $Y$ and $X$ are assumed to be ignorable. The results shown in Table 3 provide little support for the proposition that the probability of success varies with renin response. The $95 \%$ confidence intervals for $\Pi_{t}\left(x_{j}\right)-\Pi_{t}\left(x_{k}\right)$ contain zero even when only complete-data records are used.

\section{AN ILLUSTRATIVE APPLICATION TO A QUASI-EXPERIMENT}

Social scientists have long sought to understand how family structure during childhood affects childrens' outcomes later in life. Experiments in which family structure is purposefully randomized across children are not possible, but observational data from longitudinal surveys are often analyzed as quasiexperiments in which statistical associations between family structure and children's outcomes are interpreted as estimates of treatment effects. Such quasi-experimental studies have usually analyzed only cases with complete covariate and outcome data, thus effectively assuming that missing data are ignorable. See, for example, Astone and McLanahan (1991), Hogan and Kitagawa (1985), Krein and Beller (1988), and McLanahan and Sandefur (1994).

Manski, et al. (1992) used data from the National Longitudinal Study of Youth (NLSY) initiated in 1979 to estimate the effect on high school graduation of living in an intact two-parent home during 
adolescence. The objective was to assess the sensitivity of inferences to assumptions about the process determining family structure. Manski, et al. (1992) did not examine the sensitivity of their inferences to assumptions about the process generating missing data. Relying on the findings of Sandefur, McLanahan, and Wojtkiewicz (1989), they analyzed only cases with complete data. The results in Section 2 can be used to assess the identification problem posed by missing data in the quasi-experiment in which family structure is assumed to be randomly assigned conditional on certain covariates. To illustrate, we reexamine the finding of Manski, et al. (1992, p. 35) that the effect of family structure on high school graduation tends to decrease with parents' schooling.

We focus on the 1578 female NLSY respondents born in the years 1961-1964 whose race/ethnicity is neither black nor Hispanic. Family structure is a binary treatment, with $t=1$ if the respondent lived with both parents at age 14, and $t=2$ otherwise. The outcome variable $Y=1$ if the respondent received a high school diploma or GED certificate by 1985. $Y=0$ otherwise. The covariates measure parents' schooling. They are mother's $\left(X_{1}\right)$ and father's $\left(X_{2}\right)$ years of schooling in 1979. They are coded here as less than 12 , 12, and greater than 12 years of schooling. The data on birth year, sex, race/ethnicity, and family structure are complete, but there are missing values of $Y, X_{1}$ and $X_{2}$. The pattern of missingness is as follows:

$\begin{array}{ccccccccc}\text { Treatment } & \text { None } & \begin{array}{c}\text { Only } \\ \mathrm{Y}\end{array} & \begin{array}{c}\text { Only } \\ \mathrm{X}_{1}\end{array} & \begin{array}{c}\text { Only } \\ \mathrm{X}_{2}\end{array} & \begin{array}{c}\text { Missing } \\ \text { Only } \\ \left(\mathrm{Y}, \mathrm{X}_{1}\right)\end{array} & \begin{array}{c}\text { Only } \\ \left(\mathrm{Y}, \mathrm{X}_{2}\right)\end{array} & \begin{array}{c}\text { Only } \\ \left(\mathrm{X}_{1}, \mathrm{X}_{2}\right)\end{array} & \left(\mathrm{Y}, \mathrm{X}_{1}, \mathrm{X}_{2}\right) \\ 1 & 1021 & 51 & 21 & 30 & 0 & 1 & 9 & 1 \\ 2 & 314 & 9 & 16 & 81 & 0 & 4 & 20 & 0\end{array}$

Missing outcome data are caused by attrition of respondents between 1979 and 1985. Missing covariate data are caused by item nonresponse in the 1979 baseline survey. Data are missing more often for father's than for mother's schooling, especially for respondents in non-intact families. This may reflect the fact that in the 1970s, children in single-parent homes almost always lived with their mothers.

Table 4 presents two sets of results, one using $X_{1}$ as the covariate (upper panel) and the other using $X_{2}$ (lower panel). Manski, et al. (1992) used $\left(X_{1}, X_{2}\right)$ as a two-dimensional covariate, but here we want to 
illustrate how different numbers of missing observations of $X_{1}$ and $X_{2}$ affect inference. The point estimates using only the complete cases yield the earlier study's finding that the effect of family structure on high school graduation decreases with parents' schooling. The hypothesis that the effects of family structure are the same with $X_{1}<12$ and $X_{1}>12$ can be rejected at the 0.05 level. However, the hypothesis that treatment effect is independent of $X_{2}$ cannot be rejected at the 0.05 level. The estimates assuming that $X_{1}$ is MCAR continue to indicate that the effect of family structure decreases as $X_{1}$ increases. With no assumptions on the distribution of missing data, the point estimates weakly suggest that the effect may decrease with increasing $X_{1}$. The confidence intervals are very wide however, and it is not possible to reject the hypothesis that the effect of family structure is independent of $X_{1}$.

\section{CONCLUSION}

Inference from clinical trials or quasi-experiments is problematic when some data are missing because population parameters are not identified unless one makes untestable assumptions about the distribution of the missing data. This paper has shown how population parameters can be bounded without making untestable distributional assumptions. The bounds are sharp; that is, they exhaust all of the information available from the data. Two illustrative applications have demonstrated the sensitivity of substantive conclusions to the assumptions that are made about the process that generates missing data.

\section{APPENDIX: PROOFS OF THEOREMS}

Proof of Theorem 1: To obtain the sharp lower bound on $\Pi_{t}(x)$, minimize the right-hand side of (2) over $Q_{t 00}(x) \in[0,1], E_{t j 0}(x) \in[0,1](j=0,1)$, and $E_{t 01}(x)$ satisfying (4) while holding $Q_{t 01}(x)$ fixed. This yields 


$$
\begin{aligned}
\Pi_{t}(x) & \geq E_{t 11}(x) \frac{Q_{t 11}(x) p_{t 11}}{\sum_{j} Q_{t 1 j}(x) p_{t 1 j}+p_{t 00}+Q_{t 01}(x) p_{t 01}} \\
& +\max \left[0, \frac{A_{t 01}-1+Q_{t 01}(x)}{Q_{t 01}(x)}\right] \frac{Q_{t 01}(x) p_{t 01}}{\sum_{j} Q_{t 1 j}(x) p_{t 1 j}+p_{t 00}+Q_{t 01}(x) p_{t 01}} .
\end{aligned}
$$

Now minimize the right-hand side of (A.1) with respect to $Q_{t 01}(x) \in[0,1]$. This can be done by observing that the minima with respect to $Q_{t 01}(x) \in\left[0,1-A_{t 01}\right]$ and $Q_{t 01}(x) \in\left[1-A_{t 01}, 1\right]$ are both at $Q_{t 01}(x)=1-A_{t 01}$. Hence the lower bound on $\Pi_{t}(x)$ is obtained by substituting $Q_{t 01}(x)=1-A_{t 01}$ into (A.1). The upper bound is obtained by similar methods after replacing minimization operations with maximizations and observing that the maxima with respect to $Q_{t 01}(x) \in\left[0, A_{t 01}\right]$ and $Q_{t 01}(x) \in\left[A_{t 01}, 1\right]$ are both at $Q_{t 01}(x)=A_{t 01}$. Q.E.D.

Proof of Theorem 2: The upper bound is obtained through a sequence of 7 steps. STEP 1: Maximize the right-hand side of (5) with respect to $E_{t 10}\left(x_{k}\right)$ and $E_{t 00}\left(x_{k}\right)(k=1,2)$ while holding all other quantities constant. STEP 2: Maximize the result of Step 1 with respect to $Q_{t 00}\left(x_{1}\right)$ subject to (8). STEP 3: Maximize the result of Step 2 with respect to $E_{t 01}\left(x_{2}\right)$ subject to (9). STEP 4: Maximize the result of Step 3 with respect to $Q_{t 01}\left(x_{2}\right)$ subject to (7) and (10). STEP 5: Maximize the result of Step 4 with respect to $E_{t 01}\left(x_{1}\right)$. STEP 6: Maximize the result of Step 5 with respect to $Q_{t 01}\left(x_{1}\right)$ subject to (7) and (10). STEP 7: Maximize the result of Step 6 with respect to $Q_{t 00}\left(x_{2}\right)$ subject to $0 \leq Q_{t 00}\left(x_{2}\right) \leq 1$. The lower bound is minus the upper bound on $\Pi_{t}\left(x_{1}\right)-\Pi_{t}\left(x_{2}\right)$. Q.E.D.

Proof of Lemma 1: Only part (a) is proved. The proof of part (b) is similar. Part (a) asserts that $E_{t 1 k}(x)=E_{t 0 k}(x)$ for $k=0$ or 1 . To prove this, observe that 


$$
\begin{aligned}
E_{t j k}(x) & =\frac{P_{t}\left(Y=1, X=x, Z_{x}=j, Z_{y}=k\right)}{P_{t}\left(X=x, Z_{x}=j, Z_{y}=k\right)} \\
& =\frac{P_{t}\left(Z_{x}=j \mid Y=1, X=x, Z_{y}=k\right) P_{t}\left(Y=1, X=x, Z_{y}=k\right)}{P_{t}\left(Z_{x}=j \mid X=x, Z_{y}=k\right) P_{t}\left(X=x, Z_{y}=k\right)} \\
= & \frac{P_{t}\left(Z_{x}=j\right) P_{t}\left(Y=1, X=x, Z_{y}=k\right)}{P_{t}\left(Z_{x}=j\right) P_{t}\left(X=x, Z_{y}=k\right)} \\
= & P_{t}\left(Y=1 \mid X=x, Z_{y}=k\right) .
\end{aligned}
$$

Therefore, $E_{t j k}(x)$ is independent of $j$. Q.E.D.

Proof of Theorem 3: The bounds on $\Pi_{t}(x)$ are obtained by maximizing and minimizing the righthand side of (13) with respect to $E_{t 10}(x) \in[0,1]$. To obtain the bounds on $\Pi_{t}(x)-\Pi_{s}(x)$, observe that (13) holds for each treatment. Now maximize and minimize the difference between the two versions of (13) with respect to $E_{t 10}(x)$ and $E_{s 10}(x)$. Q.E.D.

Proof of Theorem 4: Equation (13) holds for $X=x_{1}$ and $X=x_{2}$. Therefore,

$$
\begin{aligned}
\Pi_{t}\left(x_{2}\right)-\Pi_{t}\left(x_{1}\right) & =E_{t 11}\left(x_{2}\right) \frac{Q_{t 11}\left(x_{2}\right)\left(p_{t 11}+p_{t 01}\right)}{\sum_{i, j} Q_{t 1 j}\left(x_{2}\right) p_{t i j}}+E_{t 10}\left(x_{2}\right)\left[1-\frac{Q_{t 11}\left(x_{2}\right)\left(p_{t 11}+p_{t 01}\right)}{\sum_{i, j} Q_{t 1 j}\left(x_{2}\right) p_{t i j}}\right] \\
& -E_{t 11}\left(x_{1}\right) \frac{Q_{t 11}\left(x_{1}\right)\left(p_{t 11}+p_{t 01}\right)}{\sum_{i, j} Q_{t 1 j}\left(x_{1}\right) p_{t i j}}-E_{t 10}\left(x_{1}\right)\left[1-\frac{Q_{t 11}\left(x_{1}\right)\left(p_{t 11}+p_{t 01}\right)}{\sum_{i, j} Q_{t 1 j}\left(x_{1}\right) p_{t i j}}\right] .
\end{aligned}
$$

$E_{t 10}\left(x_{2}\right)$ and $E_{t 10}\left(x_{1}\right)$ are the only quantities on the right-hand side of (A.2) that are not identified by the sampling process. The right hand side of (A.2) is maximized by setting $E_{t 10}\left(x_{2}\right)=1$ and $E_{t 10}\left(x_{1}\right)=0$, which gives the upper bound in the theorem. The right-hand side of (A.2) is minimized by setting $E_{t 10}\left(x_{2}\right)=0$ and $E_{t 10}\left(x_{1}\right)=1$, which gives the lower bound. Q.E.D. 


\section{REFERENCES}

Angrist, J., G. Imbens, and D. Rubin (1996), "Identification of Causal Effects Using Instrumental Variables," Journal of the American Statistical Association, 91, 444-455

Astone, N. M. and McLanahan, S. (1991), "Family Structure, Parental Practices, and High School Completion," American Sociological Review, 56, 309-320.

Balke, A. and J. Pearl (1997), "Bounds on Treatment Effects from Studies With Imperfect Compliance," Journal of the American Statistical Association, 92, 1171-1177.

Bickel, P.J. and Freedman, D.A. (1981), "Some Asymptotic Theory for the Bootstrap," Annals of Statistics, 9, 1196-1217.

Bloom, H. (1984), "Accounting for No-Shows in Experimental Evaluation Designs," Evaluation Review, $8,225-246$.

Freis, E.D., Materson, B.J., and Flamenbaum, W. (1983), "Comparison of Propranolol or Hydorchlorothiazide Alone for Treatment of Hypertension, III: Evaluation of the Renin-Angiotensin System," The American Journal of Medicine, 74, 1029-1041.

Hogan, D. P. and Kitagawa, E. M. (1985), "The Impact of Social Status, Family Structure, and Neighborhood on the Fertility of Black Adolescents," American Journal of Sociology, 90, 825-855.

Horowitz, J.L. and Manski, C.F. (1995), "Identification and Robustness with Contaminated and Corrupted Data," Econometrica, 63, 281-302.

Horowitz, J.L. and Manski, C.F. (1998), "Censoring of Outcomes and Regressors Due to Survey Nonresponse: Identification and Estimation Using Weights and Imputations," Journal of Econometrics, 84, 37-58.

Krein, S. F. and Beller, A. H. (1988), "Educational Attainment of Children from Single-Parent Families: Differences by Exposure, Gender, and Race," Demography, 25, 221-234.

Little, R. (1992), "Regression with Missing X's: A Review," Journal of the American Statistical Association, 87, 1227-1237.

Little, R. (1995), "Modeling the Drop-Out Mechanism in Repeated-Measures Studies," Journal of the American Statistical Association, 90, 1112-1121.

Little, R. and Yau, L. (1996), "Intent-to-Treat Analysis for Longitudinal Studies with Drop-Outs," Biometrics, 32, 1324-1333.

Manski, C. F. (1989), “Anatomy of the Selection Problem,” Journal of Human Resources, 24, 343-360.

Manski, C. F. (1990), "Nonparametric Bounds on Treatment Effects," American Economic Review Papers and Proceedings, 80, 319-323. 
Manski, C.F. (1995), Identification Problems in the Social Sciences, Cambridge, MA: Harvard University Press.

Manski, C. F. (1997), "The Mixing Problem in Programme Evaluation,” Review of Economic Studies, 64, 537-553.

Manski, C. F., Sandefur, G. D., McLanahan, S, and Powers, D. (1992), "Alternative Estimates of the Effect of Family Structure During Adolescence on High School Graduation," Journal of the American Statistical Association, 87, 25-37.

Materson, B.J., Reda, D.J., Cushman, W.C., Massie, B.M., Freis, E.D., Kochar, M.S., Hamburger, R.J., Fye, C., Lakshman, R., Gottdiener, J., Ramirez, E.A., and Henderson, W.G. (1993), "Single-Drug Therapy for Hypertension in Men: A Comparison of Six Antihypertensive Agents with Placebo," The New England Journal of Medicine, 328, 914-921.

Materson, B.J. and Reda, D.J. (1994), "Correction: Single-Drug Therapy for Hypertension in Men," The New England Journal of Medicine, 330, 1689.

Materson, B.J., Reda, D.J. and Cushman, W.C. (1995). "Department of Veterans Affairs Single-Drug Therapy of Hypertension Study: Revised Figures and New Data," American Journal of Hypertension, 8, 189-192.

McLanahan, S. and Sandefur, G. D. (1994), Uncertain Childhood, Uncertain Future, Cambridge, MA: Harvard University Press.

Nordheim, E.V. (1984), "Inference from Nonrandomly Missing Categorical Data: An Example from a Genetic Study on Turner's Syndrome," Journal of the American Statistical Association, 79, 772-780.

Robins, J. (1989), "The Analysis of Randomized and Non-Randomized AIDS Treatment Trials Using a New Approach to Causal Inference in Longitudinal Studies," in Sechrest, L., H. Freeman, and A. Mulley. eds. Health Service Research Methodology: A Focus on AIDS, NCHSR, U.S. Public Health Service.

Robins, J., Rotnitzky, A., and Zhao, L. (1994), "Estimation of Regression Coefficients When Some Regressors Are Not Always Observed," Journal of the American Statistical Association, 89, 846-866.

Rosenbaum, P. R. (1995), Observational Studies, New York: Springer-Verlag.

Rotnitsky, A., Robins, J.M., and Scharfstein, D. O. (1998), 'Semiparametric Regression for Repeated Outcomes with Nonignorable Nonresponse," Journal of the American Statistical Association, 93, 13211339.

Sandefur, G. D., McLanahan, S. and Wojtkiewicz, R. A. (1989), "Race, Ethnicity, Family Structure, and High School Graduation," Madison, Wisconsin: Institute for Research on Poverty Discussion Paper \#893-89.

Wang, C., Wang, S., Zhao, L. and Ou, S. (1997), "Weighted Semiparametric Estimation in Regression Analysis with Missing Covariate Data," Journal of the American Statistical Association, 92, 512-525. 
Table 1: Pattern of Missingness in the DVA Data

\begin{tabular}{ccccccc} 
Treatment & $\begin{array}{c}\text { Number } \\
\text { Randomized }\end{array}$ & $\begin{array}{c}\text { Observed } \\
\text { Successes }\end{array}$ & $\begin{array}{c}\text { None } \\
\text { Missing }\end{array}$ & $\begin{array}{c}\text { Missing } \\
\text { Only Y }\end{array}$ & $\begin{array}{c}\text { Missing } \\
\text { Only X }\end{array}$ & \multicolumn{2}{c}{$\begin{array}{c}\text { Missing } \\
\text { Y and X }\end{array}$} \\
1 & 188 & 100 & 173 & 4 & 11 & 0 \\
3 & 178 & 106 & 158 & 11 & 9 & 0 \\
4 & 188 & 96 & 169 & 6 & 13 & 0 \\
5 & 178 & 110 & 159 & 5 & 13 & 1 \\
6 & 185 & 130 & 164 & 6 & 14 & 1 \\
7 & 188 & 97 & 164 & 12 & 10 & 2 \\
& 187 & 57 & 178 & 3 & 6 & 0
\end{tabular}


Table 2: Bounds on $\Pi_{t}(x)-\Pi_{7}(x)$ for DVA Clinical Trial ${ }^{a}$

No Assumptions about Missing Data

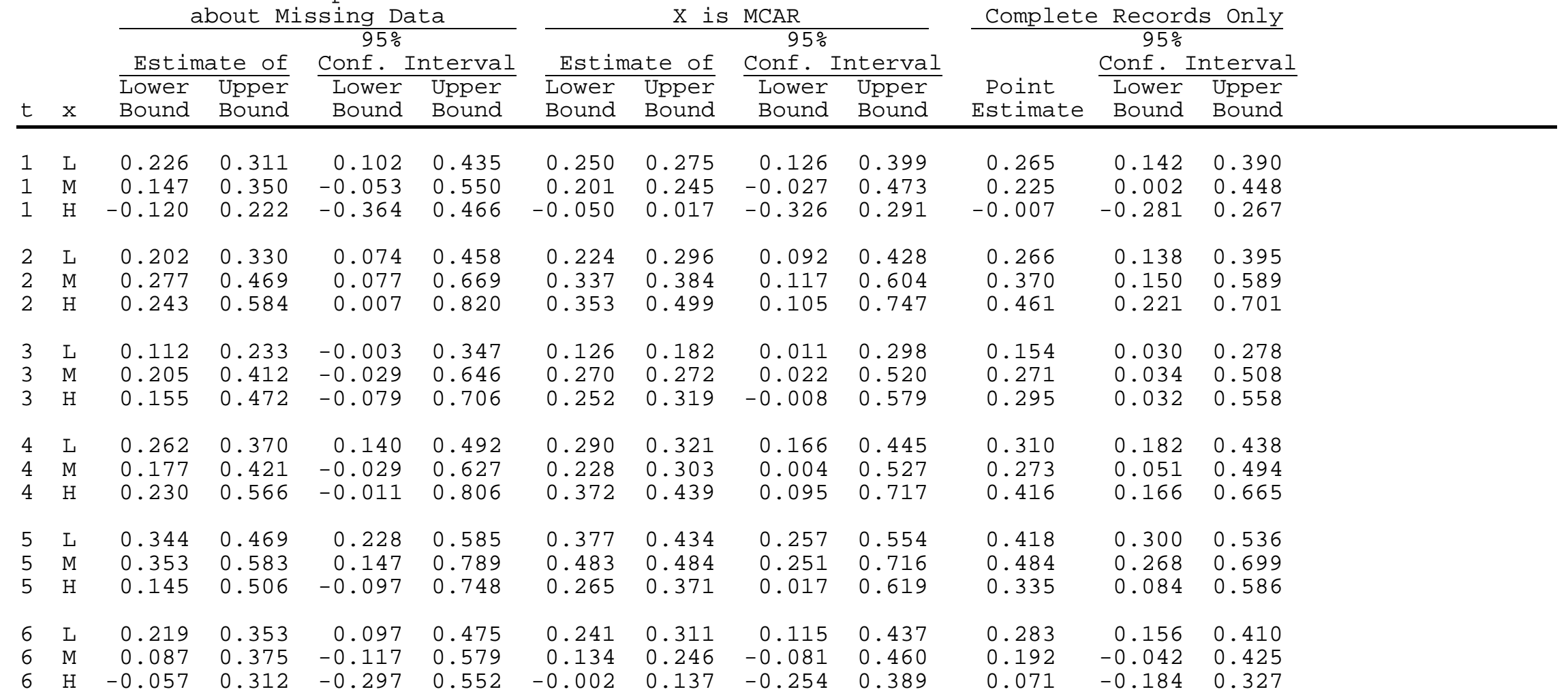

a With no assumptions about missing data and the assumption that $X$ is MCAR, the confidence ingerval is obtained with the bootstrap and contains the population lower and upper bounds with asymptotic probability 0.95. With complete records only, the confidence interval is obtained by the delta method. 
Table 3: Bounds on $\Pi_{t}\left(x_{2}\right)-\Pi_{t}\left(x_{1}\right)$ for DVA Clinical Trial

No Assumptions

about Missing Dat

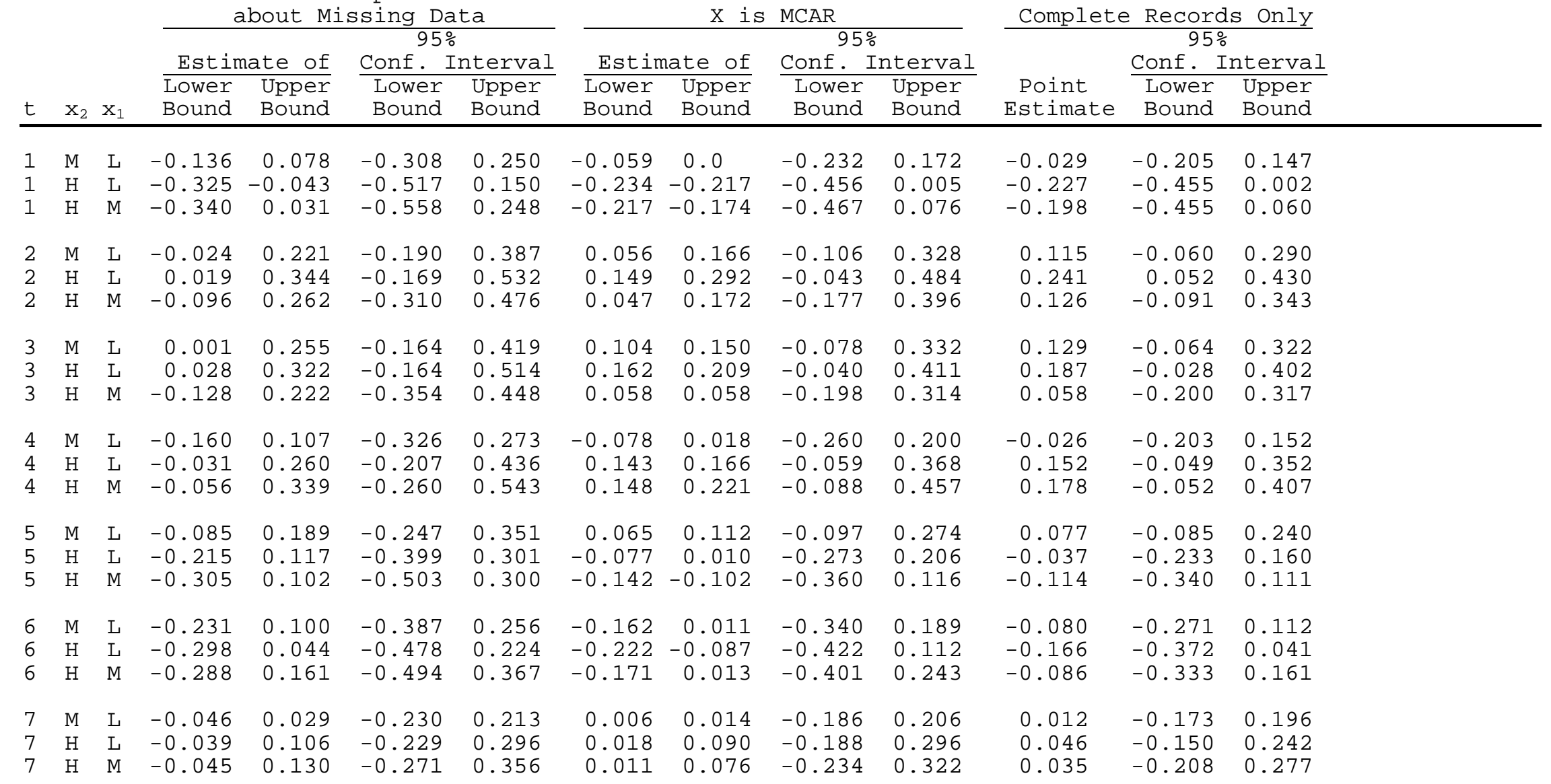


Table 4: Bounds on $\Pi_{1}(\mathrm{x})$ - $\Pi_{2}(\mathrm{x})$ for Analysis of Effects of Family structure

No Assumptions

about Missing Data

$$
\text { 95\% }
$$

Estimate of Conf. Interval

Lower Upper Lower Upper

$\mathrm{X}$ is MCAR

95\%

Estimate of Conf. Interval

Lower Upper Lower Upper

Complete Records Only

$\mathrm{X}$

Bound Bound

Bound Bound

Bound Bound

Bound Bound

Point Conf. Interval

Estimate Bound Bound

$\mathrm{X}$ is mother's schooling

\begin{tabular}{|c|c|c|c|c|c|c|c|c|c|c|c|}
\hline $\begin{array}{r}<12 \\
12 \\
>12\end{array}$ & $\begin{array}{r}0.226 \\
0.049 \\
-0.058\end{array}$ & $\begin{array}{l}0.311 \\
0.225 \\
0.215\end{array}$ & $\begin{array}{r}0.026 \\
-0.019 \\
-0.146\end{array}$ & $\begin{array}{l}0.389 \\
0.293 \\
0.303\end{array}$ & $\begin{array}{r}0.173 \\
0.087 \\
-0.011\end{array}$ & $\begin{array}{l}0.262 \\
0.165 \\
0.039\end{array}$ & $\begin{array}{r}0.095 \\
0.017 \\
-0.081\end{array}$ & $\begin{array}{l}0.340 \\
0.235 \\
0.109\end{array}$ & $\begin{array}{r}0.230 \\
0.139 \\
-0.201\end{array}$ & $\begin{array}{r}0.143 \\
0.072 \\
-0.042\end{array}$ & $\begin{array}{l}0.317 \\
0.206 \\
0.085\end{array}$ \\
\hline & & & & & & $x$ is & SWIEd & 年 & & & \\
\hline $\begin{array}{r}<12 \\
12 \\
>12\end{array}$ & $\begin{array}{r}-0.023 \\
0.018 \\
-0.044\end{array}$ & $\begin{array}{l}0.352 \\
0.370 \\
0.460\end{array}$ & $\begin{array}{l}-0.016 \\
-0.054 \\
-0.132\end{array}$ & $\begin{array}{l}0.434 \\
0.442 \\
0.547\end{array}$ & $\begin{array}{l}0.126 \\
0.117 \\
0.039\end{array}$ & $\begin{array}{l}0.207 \\
0.193 \\
0.116\end{array}$ & $\begin{array}{r}0.036 \\
0.037 \\
-0.051\end{array}$ & $\begin{array}{l}0.297 \\
0.273 \\
0.206\end{array}$ & $\begin{array}{l}0.176 \\
0.170 \\
0.082\end{array}$ & $\begin{array}{r}0.082 \\
0.092 \\
-0.002\end{array}$ & $\begin{array}{l}0.271 \\
0.249 \\
0.166\end{array}$ \\
\hline
\end{tabular}

